\title{
Cipango
}

Cahiers d'études japonaises

$24 \mid 2021$

Eugénisme dans le Japon moderne et contemporain

BOUCHY Anne (dir.), 2013, Le vivre ensemble à Sasaguri, une commune de Kyūshū. Dans l'entrelacs des dynamiques du dedans et du dehors - Études d'ethnologie du Japon, Cahiers d'Extrême-Asie 22, EFEO, Kyōto, 650 p.

\section{Carina Roth}

\section{OpenEdition}

\section{Journals}

Édition électronique

URL : https://journals.openedition.org/cipango/5098

DOI : $10.4000 /$ cipango.5098

ISSN : 2260-7706

Éditeur

INALCO

Édition imprimée

Date de publication : 20 octobre 2021

Pagination : 319-327

ISBN : 9782858313969

ISSN : 1164-5857

Référence électronique

Carina Roth, « воucHy Anne (dir.), 2013, Le vivre ensemble à Sasaguri, une commune de Kyūshū. Dans l'entrelacs des dynamiques du dedans et du dehors - Études d'ethnologie du Japon, Cahiers d'ExtrêmeAsie 22, EFEO, Kyōto, 650 p. », Cipango [En ligne], 24 | 2021, mis en ligne le 11 octobre 2021, consulté le 10 avril 2022. URL : http://journals.openedition.org/cipango/5098; DOI : https://doi.org/10.4000/ cipango.5098

\section{(c) (†) (8)}

Cipango est mis à disposition selon les termes de la Licence Creative Commons Attribution - Pas d'Utilisation Commerciale 4.0 International. 


\section{Bouchy Anne (dir.), 2013, Le vivre ensemble à Sasaguri, une commune de Kyüshü. Dans l'entrelacs des dynamiques du dedans et du dehors - Études d'ethnologie du Japon, Cahiers d'Extrême-Asie 22, EFEO, Kyōto, 650 p.}

Carina Roth

UCSB

Ce volumineux numéro spécial des Cahiers d'Extrême-Asie, publiés par le centre de l'École française d'Extrême-Orient (EFEO) à Kyōto, est le résultat d'un impressionnant travail collectif qui s'est étendu sur plusieurs années, entre 2004 et 2010. Il est entièrement consacré à l'étude multilatérale et transdisciplinaire d'une commune du nord de Kyūshū, Sasaguri 篠栗. Si l'accent est mis sur les aspects religieux de ce site de pèlerinage, chacun des articles de l'ouvrage met en lumière des éléments relevant du social, de l'économique, du politique, de l'environnemental, de façon à rendre une image aussi inclusive et cohérente que possible de la vie d'une communauté contemporaine dans toute sa complexité.

Outre une présentation de synthèse en début de volume, l'ouvrage compte huit essais d'envergure, puisque les articles comptent entre 50 et 100 pages chacun. Six des huit articles sont traduits du japonais. Il s'agit d'un projet aussi ambitieux que novateur à divers égards : c'est la première publication de cet ordre en français, associant en une étroite collaboration des enseignants-chercheurs français et japonais et leurs étudiants, les habitants de Sasaguri, et le Centre local 


\section{CIPANGO \\ 320 Cahiers d'études japonaises nº 24}

de documentation de la municipalité. Enfin, le volume doit encore être complété par une base de données regroupant archives et autres documents, qui sera mise à disposition sur le site de l'EFEO.

Comme l'explique Anne Bouchy, qui a réalisé l'immense travail d'édition que représente un projet de cette ampleur, tout comme la quasi-totalité des traductions, le volume est le fruit d'une réflexion ethnologique partagée, et appuyée sur une ethnologie collective, fondée sur six enquêtes annuelles s'étendant de dix jours à trois semaines. Le projet est issu de l'un des programmes francojaponais de recherche quadriennaux de l'EFEO («Entre "dedans" et "dehors" : les dynamiques socioculturelles au Japon »). Il inclut la participation de chercheurs universitaires, de leurs étudiants et des habitants de Sasaguri. Le choix de cette commune repose sur un dénominateur d'intérêt commun aux chercheurs associés au projet : que ce dernier soit centré sur les aspects religieux d'un lieu donné. Sasaguri, avec son circuit répliqué des 88 temples de Shikoku et sa renommée de 《région de capacités subtiles 》 (reinō chitai 霊能地帯) représente un site d'observation idéal. La cohésion interne du projet, déjà solidement assise par le fait que l'ensemble des articles porte sur un même lieu géographique relativement restreint, est doublée d'une réflexion appuyée sur les dynamiques relationnelles se mettant en place entre le dedans et le dehors sous tous leurs aspects. Loin d'être considérés comme des catégories étanches les unes aux autres, ces deux principes, perçus comme complémentaires, non exclusifs et surtout inséparables (p. 7), sont employés comme outil d'analyse permettant de ramener tout objet d'étude à une mesure commune.

Le volume est divisé en trois parties correspondant à des approches différentes. Les deux premiers essais plantent le « cadre socio-culturel, historique et environnemental ». Suzuki Masataka, dans le premier de deux articles, examine la forte identité communautaire de Wakasugi 若杉山, la municipalité dans laquelle est située l'un des deux sites religieux les plus importants de Sasaguri, le sanctuaire de Taiso 太祖宮. Anne Bouchy, dans l'essai suivant, décrit les montagnes-forêts et les rapports intercommunautaires à l'environnement à Sasaguri. Le deuxième volet du projet est consacré aux différentes « textures du religieux ». Mori Hiroko analyse l'histoire du culte du sanctuaire de Taiso, sur le Mont Wakasugi, puis Nakayama Kazuhisa celle de la réplique du pèlerinage des quatre-vingt-huit temples de Shikoku, qui représente l'attraction principale du 
site de Sasaguri. Dans un second article de sa plume, Suzuki Masataka se penche sur le Nanzō-in 南蔵院, le temple ouvrant le circuit de pèlerinage de Sasaguri, et les différentes stratégies mises en œuvre par ses supérieurs successifs pour attirer le public. Charlotte Lamotte clôt cette section par un essai sur la dimension de l'individu et de sa relation au religieux à travers l'examen des liens construits entre fidèles et les innombrables statues du parcours de Sasaguri. La dernière approche est consacrée à la vie des maisons (ie) et des femmes. Ishikawa Toshiko propose une étude de l'évolution de la maison en tant que foyer familial et lieu de transmission générationnelle sur le plan symbolique et religieux. Kanda Yoriko, quant à elle, dresse une pluralité de portraits de femmes, présentés par le biais d'une ethnographie narrative. Cette dernière donne une image vivante de deux strates différentes de la population féminine de Sasaguri, celle liée au monde des temples, et à celui des mines.

\section{Cadre socioculturel et environnemental}

Suzuki Masataka 鈴木正崇： «Continuités et transformations de la société locale - Le fait coutumier dans le village de Wakasugi (Sasaguri, Fukuoka) », p. 27-113 (trad. Anne Bouchy).

Ce premier article retrace l'histoire du village de Wakasugi du point de vue de l'anthropologie culturelle. Sur la base d'engi 縁起 (histoires des origines) rédigés entre le $\mathrm{XVI}^{\mathrm{e}}$ et le début du $\mathrm{xx}^{\mathrm{e}}$ siècle, Suzuki retrace dans un premier temps le « processus d'historicisation, de transposition d'une histoire fictive en histoire authentique » (p. 45) à l'œuvre dans ce type de textes. Il met en lumière les éléments de continuité, comme la sacralisation de la montagne et des eaux, l'intégration au bouddhisme du lieu d'origine, les rapports à l'étranger comme à la société locale. En contrepoint, il démontre les changements structurels des différents établissements religieux de la commune en fonction des changements sociaux et politiques, en particulier à l'époque Meiji, et jusqu'à aujourd'hui. Au travers de l'examen des engi et chroniques, de la reconstruction de festivals et ritualités après Meiji, et des transformations dues à la modernisation de la société, cette étude montre la mobilité et la malléabilité de la transmission culturelle d'une communauté locale sur le long terme. 
CIPANGO

322 Cahiers d'études japonaises nº 24

Anne Bouchy : «Les rapports communautaires aux espaces forestiers entre politiques du dehors et stratégies du dedans : les montagnes-forêts de Sasaguri », p. 115-201.

Le cadre général de cette étude est une réflexion sur les espaces montagneux et forestiers, et leur rôle changeant dans la société villageoise de Sasaguri, mais aussi au-delà. Le point focal de l'essai est la notion de «montagne du village » (sato yama 里山), et l'usage des « montagnes-forêts 》 (yama 山) comme ressources. En quelques décennies, ces espaces limitrophes des communautés habitées ont perdu leur rôle de réservoirs de matières premières (fourrage, engrais, combustible, bois de construction, produits de subsistance, pharmacopée, etc.). En décomposant la part de « nature » et celle « d'héritage culturel » par rapport au sato yama, Bouchy offre un point de vue intégrant les aspects économiques, écologiques, sociaux et culturels d'une communauté. À ce titre, sont examinées plusieurs configurations locales de gestion pour les espaces forestiers (coopératives et gestion communautaire). L'étude se conclut sur une nouvelle direction de développement des espaces forestiers, celle de la « thérapie forestière 》(foresuto serapii フォレス トセラピー) en tant que 《 forme la plus actuelle de la socialisation des forêts » (p. 191). Prenant appui sur le cadre local de Sasaguri, cette étude démontre que les forêts, paradigme même de la «nature » a priori, sont en réalité des espaces hautement anthropisés dans leur déclinaison villageoise.

\section{Texture du religieux}

Mori Hiroko 森弘子 : «Le mont Wakasugi, son sanctuaire et le shugen dans l'histoire de Kyūshü et de l'Asie de l'Est », p. 203-267 (trad. Anne Bouchy).

L'étude de Mori repose sur un dépouillement minutieux des archives de l'Ishii-bō 石井坊, à partir desquelles elle remonte l'histoire cultuelle de Wakasugi d'un côté, et de l'autre, éclaire le rôle de l'Ishii-bô dans le contexte régional. La première moitié de l'essai se concentre sur les liens entre le Mont Wakasugi et les cultes de proximité régionale directe (Munakata 宗像et de Hachiman 八幡), ainsi qu'avec ceux du shugen 修験 de Kumano 熊野, expliquant leur interaction et fusion partielle. Sur la base des archives de l'Ishii-bō, la seconde partie est consacrée à la 
description et l'analyse des rites et entrées en montagne, des liens entre yamabushi 山伏 (l'un des noms donnés aux pratiquants shugen) de montagne et yamabushi de village, ainsi qu'aux interactions entre fief et organisation(s) shugen au niveau local, régional et suprarégional. L'essentiel des sources porte sur fin Edo, avant la Restauration de Meiji. La richesse des archives permet à l'auteur de donner une image vivante des rapports de force entre diverses organisations sociales, politiques et religieuses.

Nakayama Kazuhisa 中山和久 : «La dynamique de création, réplication et déclin des lieux de pèlerinage - Le nouveau pèlerinage de Shikoku à Sasaguri », p. 269-350 (trad. Anne Bouchy).

Depuis sa création au milieu du $\mathrm{XIX}^{\mathrm{e}}$ siècle, le nouveau pèlerinage de Shikoku à Sasaguri est l'attraction principale du site. Les premières répliques de pèlerinage sont attestées dès le $\mathrm{XII}^{\mathrm{e}}$ siècle, mais la grande popularité du culte de Kōboō daishi 弘法大師 au XIX ${ }^{\mathrm{e}}$ siècle entraîne la prolifération à travers tout le Japon de répliques des quatre-vingt-huit stations du pèlerinage de Shikoku. Le circuit de Sasaguri, fondé en 1835 par la nonne Ji.in 慈忍 et achevé en 1855, se développe en corrélation avec l'expansion économique et commerciale de la région. Celle-ci est due à l'essor des houillères et la demande exponentielle en charbon dans le contexte de la politique de renforcement économique et militaire du pays à la fin $\mathrm{du} \mathrm{XIX}^{\mathrm{e}}$ siècle. L'étude examine le développement du pèlerinage sous l'angle de quatre dynamiques différentes : dynamique de création du pèlerinage (genèse); dynamique de la genèse du pèlerin (réception) ; dynamique de transformation du pèlerin par les lieux de pèlerinage (action); dynamique de transformation des lieux de pèlerinage par les pèlerins (re-création).

Suzuki Masataka : «Modernisation des temples bouddhiques et société locale Le Nanzō-in de Sasaguri », p. 351-421 (trad. Ignacio Quiros, Iyanaga Nobumi, Anne Bouchy).

En retraçant l'histoire du Nanzō-in à travers les générations successives de ses supérieurs, Suzuki trace le portrait non seulement de l'évolution d'un temple bouddhique donné et des besoins spirituels auxquels il répond, mais aussi de la 
manière dont la personnalité des moines qui la dirige infléchit le développement d'une institution religieuse. Dans le cas du Nanzō-in, les quatre supérieurs qui ont traversé le $\mathrm{XX}^{\mathrm{e}}$ siècle jusqu'à aujourd'hui sont des personnalités particulièrement dynamiques, qui ont chacune proposé des solutions aussi ingénieuses que variées pour assurer la survie et le développement de leur temple. Le Nanzō-in est le point de départ et temple faîtier des quatre-vingt-huit fudasho 札所 (《lieux où l'on dépose les vignettes ») du pèlerinage de Sasaguri. Afin de contrer le déclin du pèlerinage à partir des années 1990, Hayashi Kakujō 林覚乗, supérieur du Nanzō-in depuis 1980, a transformé le site d'un temple spécialisé dans les rites à la demande (kitō-dera 祈䘠寺) en un centre de rites funéraires et d'offices pour les ancêtres. À cette fin, il a fait construire un premier, puis un second columbarium, et surtout une statue de Bouddha couché de 41 mètres, inaugurée en 1995 et dite être la plus grande statue de bronze au monde. Suzuki démontre comment le Nanzō-in (et Sasaguri dans son sillage), par le biais de l'inventivité et du charisme de son supérieur actuel, est aussi un exemple frappant du caractère hybride du bouddhisme japonais, cumulant et combinant des traditions aux origines diverses, faisant de Sasaguri un « lieu d'expérimentation dans les affaires funéraires », car plusieurs autres temples ont suivi le mouvement (p. 378).

\section{Charlotte Lamotte : «La pierre qui vit - Naissance et mort des statues dans une ville de pèlerinage (Sasaguri, Fukuoka, Japon) », p. 427-472.}

Dans cet essai, Lamotte explore les différents modes par lesquels un dialogue se met en place entre un objet matériel considéré comme sacré et une personne venant se recueillir devant lui. L'interrogation dont est issu ce travail provient d'une remarque faite à l'auteur par l'un de ses interlocuteurs : « même si les deux statues ont été faites par le même sculpteur et sur le même modèle, celle-là est belle, alors que l'autre est quelconque » (p. 424). L'objectif de la recherche est d'examiner les rapports entre les objets et les hommes qui les vénèrent, ainsi que les interactions permanentes unissant fidèles et objets de culte. À chaque statue des quatre-vingt-huit stations du pèlerinage de Sasaguri se sont jointes une multitude d'autres statues, souvent des ensembles de statues, si bien que le site se prête particulièrement bien à une réflexion de ce type. Lamotte relève les différentes modalités d'interaction entre statues et fidèles, moines, sculpteurs, officiants, 
etc. Toutes ont pour dénominateur commun la transmission d'une part affective de la personne dans la statue, qui devient réceptacle tant d'une présence divine, ou appartenant à l'au-delà, que d'une présence humaine. Outre une explication détaillée des rites «d'ouverture des yeux » (kaigen shiki 開眼式) et de « retrait de l'âme » (tama nuki 霊抜き) par lesquels une statue, une image ou un objet bouddhique, sont transformés en icônes, Lamotte rapporte également qu'une partie de la personnalité du moine officiant passe dans la statue, de même que l'intention (nen 念) des fidèles : la statue est un objet de transfert permettant ou facilitant des échanges multivalents, par la création d'une fusion entre une entité subtile, l'objet en lui-même et les intentions des fidèles (p. 464-465).

\section{Au cour du social}

Ishikawa Toshiko 石川稔子 : «Les rites annuels de la maison et des communautés locales en transformation - Intérieur et extérieur des lieux de vie communautaires $\gg, p .473-546$.

À la différence des autres articles du volume, qui tous traitent d'espaces socioreligieux extérieurs, cette étude examine les transformations des ritualités au sein de la maison en tant que foyer familial (ie イ工工) au cours du $\mathrm{Xx}^{\mathrm{e}}$ siècle. Ishikawa montre le passage de l'homme à la femme en tant qu'acteur rituel après-guerre, en correspondance avec la déperdition du lien entre maison et activité professionnelle. Un autre corollaire de ce changement est une tendance à l'affaiblissement de la dimension religieuse et cultuelle des rites au profit de leur perception par les participants comme une occasion de réjouissance collective (p. 500) : fête annuelle du sanctuaire tutélaire, fête du dieu des grains, fête des morts devenue fête de l'été à la fin des années 1980, etc. Même lorsque la finalité affichée est le culte d'une figure religieuse, la force d'attraction de l'évènement réside dans son caractère festif. Enfin, Ishikawa note un fort déplacement des ritualités de l'intérieur des maisons vers des lieux extérieurs (restaurants, sources thermales, etc.) : l'intérieur de la maison est devenu le lieu du maintien de l'ordinaire (p. 522). Les rites annuels deviennent l'occasion de réunions festives, mais sont pour l'essentiel privées de leur aspect cultuel. La banalisation du festif (p. 483) devenue monnaie courante entraîne également l'intégration de « nouvelles fêtes » (anniversaires, fêtes des 
mères, pères, etc.) au détriment des ritualités traditionnelles associant culte et repas communautaire (p. 523).

Kanda Yoriko 神田より子 : «Femmes de Sasaguri - Au fond du religieux et à l'ubac du séculier »,p. 547-606.

Le dernier essai du recueil est consacré à une série de portraits de femmes examinés sous deux angles d'approche : la relation des femmes au pèlerinage et le rôle des femmes dans les mines de charbon. Kanda passe d'abord en revue les quatre générations de femmes des supérieurs du Nanzô-in depuis le transfert de ce temple du Mont Kôya 高野山 à Sasaguri en 1899. Elle s'arrête ensuite à la personne de Shōzaki Ryōsei 庄崎良清, supérieure charismatique du Dainichi-ji 大日寺 (et mentionnée dans plusieurs des articles du volume), puis de deux autres épouses de supérieurs, avant de passer au monde des mines, avec en particulier la personnalité sulfureuse de Fujita Kototome 藤田小女姫, spécialiste de la divination, et de son lien à la prostitution. La palette est large, et la narration suit les propos des personnes interrogées, en complétant, notamment pour ce qui est des mines, le discours des femmes par des informations précises et détaillées sur le contexte historique. Cette succession de portraits ainsi que le récit de l'inscription de ces femmes dans la communauté locale et régionale est une manière à la fois belle et inattendue de clore ce vaste panorama sur Sasaguri en tant que réservoir, mais aussi laboratoire, d'activité religieuses à la jonction du pèlerinage, des rites funéraires, des pratiques charismatiques et de l'éco-spiritualité.

L'objectif de l'étude est d'une part de « mettre en lumière comment les imbrications politiques, sociales, économiques formatent et bouleversent les dimensions internes et externes d'une communauté locale » (p. 117) et d'autre part de « réfléchir aux rapports de la société au milieu naturel » (p. 119). Au bout des 650 pages du volume, ces deux objectifs sont pleinement atteints. En réalité, ce numéro spécial est un objet singulier et plaisamment anachronique par sa longueur, par la profondeur de traitement du sujet, et par le grand soin apporté à la création d'un socle commun et partagé de réflexion. Le fait que les traductions comme l'édition sont le fait, pour l'essentiel, de la même personne (Anne Bouchy), donne au volume un degré rare d'homogénéité et de cohérence. Le choix des notions de l'interne et de l'externe comme dénominateur commun 
peut paraître artificiel (cet aspect a été omis dans le présent rapport), mais par son rôle de matrice de réflexion, il a certainement contribué à la cohésion de l'ensemble. Qui plus est, en raison de la longueur même du projet, les chercheurs ont été partie tenante de cette dynamique du dedans et du dehors, devenant pour ainsi dire des « étrangers de l'intérieur » (p. 17). La longueur des articles permet une exploration des sujets sur le temps long et autorise ainsi une ampleur de réflexion difficile à rencontrer dans le cadre de recueils d'articles. Chacun des essais peut être comparé à un moyen-métrage portant sur l'un des aspects de Sasaguri en tant que site religieux d'une grande richesse et variété. Au fur et à mesure des essais, une image kaléidoscopique de ce lieu se crée, grâce au fait que les différents acteurs du site, personnes et lieux confondus, se retrouvent souvent dans plus d'un article, et sont à chaque fois présentés sous une autre facette. Le seul regret est l'absence d'une carte réellement claire et synthétique de la région, qui aurait servi de renvoi et référence à l'ensemble du volume, et aurait heureusement complété les cartes et schémas plus spécifiques inclus dans les articles. Il est impossible de rendre justice dans le cadre d'un compte-rendu à la richesse de ce volume, dont la longueur est un obstacle qu'il vaut grandement la peine de surmonter. Enfin, ce numéro spécial reflète l'intérêt croissant et bienvenu pour la région de Kyūshū dans nombre de domaines des sciences humaines. 